\title{
EXTREMAL PROPERTIES OF SOME CLASSES OF UNIVALENT FUNCTIONS
}

\section{E. NETANYAHU ${ }^{1}$}

1. Introduction. Let $S$ be the family of regular and univalent functions in the unit disk $|z|<1$ with the series development

$$
w=f(z)=z+a_{2} z^{2}+\cdots
$$

and let

$$
d_{f}=\inf \{|\alpha|: f(z) \neq \alpha,|z|<1\} .
$$

Let $S(d), 1 / 4 \leqq d \leqq 1$, be the sub-family of $S$ for which $d_{f}=d$. Because of the compactness of $S(d)$ there exists, at least one function $f(z) \in S(d)$ for which

$$
\underset{f \in S(d)}{\operatorname{Max}}\left\{\left|a_{n}\right|\right\}
$$

is attained, and we denote

$$
a_{n}(d)=\operatorname{Max}_{f \in S(d)}\left\{\left|a_{n}\right|\right\} .
$$

Consider expressions

$$
a_{n}(d) d^{\lambda}, \quad \lambda \geqq 0, \quad \frac{1}{4} \leqq d \leqq 1,
$$

and let $d_{0}$ be a local maximum for such an expression, i.e. there exists some $\eta>0$ such that

$$
a_{n}\left(d_{0}+h\right)\left(d_{0}+h\right)^{\lambda} \leqq a_{n}\left(d_{0}\right) d_{0}^{\lambda}
$$

holds for all real $h$ with $|h|<\eta$ (in case that $d_{0}=1 / 4$ only positive values of $h$ come in consideration).

There exist always points of local maxima. Indeed, for every real $\lambda$ consider the functional $\left\{\left|a_{n}\right| d^{\lambda}\right\}$ where $a_{n}$ is the $n$th coefficient of a func-

${ }^{1}$ Research partly supported by the U.S. Air Force under Grant AF-AFSOR70-1947, monitored by the Office of Scientific Research, 
tion $f(z) \in S$, and $d$ is the radius of the greatest disk around $w=0$ contained in the image of $|z|<1$ by $f(z)$. We may now ask for

$$
\operatorname{Max}_{f \in S}\left\{\left|a_{n}\right| d^{\lambda}\right\}
$$

Because of the compactness of $S$ there is, at least, one function which solves the problem. Let $d_{f}$ of the extremal function be equal to $d_{0}$. But then $\left|a_{n}\right|$ of this extremal function is equal for $a_{n}\left(d_{0}\right)$; and for this value of $d_{0}(6)$ certainly holds.

In this paper we investigate the properties of the extremal function for which the expression (5) has a local maximum at $d_{0}$. It will be shown, among other things, that $a_{n}(d)$ has at such a point a derivative from the right which is equal to $\left(-\lambda / d_{0}\right) a_{n}\left(d_{0}\right)$, and when $\lambda>0$ it has a derivative.

For $\lambda=0$ this is an additional information about the Koebe function, whose $n$th coefficient is maximal for $\left|a_{2}-2\right|<\varepsilon_{n},[1],[2]$, or when $0<d-1 / 4$ and sufficiently small.

The methods used are variational methods, and part of them were used in an earlier paper [5].

2. Characterization of an extremal function. Let $f(z)=z+$ $a_{2} z^{2}+\ldots+a_{n} z^{n}+\ldots$ be an extremal function of $S\left(d_{0}\right)$. We may assume that $a_{n}>0, a_{n}=a_{n}\left(d_{0}\right)$. Otherwise we consider the function $e^{-i \vartheta} f\left(z e^{i \vartheta}\right)$, which also belongs to $S\left(d_{0}\right)$, and choose $\vartheta$ so that the $n$th coefficient becomes real and positive.

Let $D$ be the image domain of $|z|<1$ by $f(z)$ in the $w$-plane. This domain contains the disk $|w|<d_{0}$. Consider the mapping

$$
w^{*}=w\left[1+\frac{a \varrho^{2}}{w-w_{1}}-\frac{\bar{a} \varrho^{2} w}{d_{0}^{2}-w \bar{w}_{1}}\right]
$$

where $\varrho>0,|a|=1, w_{1} \in D$ and $d^{2} / \bar{w}_{1} \in D$. We can choose $\varrho$ sufficiently small so that this mapping is regular and univalent on the boundary $C$ of $D$. The image of $C$ by (7) will be a new set of continua $C^{*}$ which bound a domain $D^{*}$, very "close" to $D$.

We observe that for $|w|=d_{0}$

$$
\left|w^{*}\right|=d_{0}\left|1+i \varrho^{2} R\right|,
$$

where $R$ is real and independent of $\varrho$. This shows that $d_{1}$, the greatest radius such that the disk $\left|w^{*}\right|<d_{1}$ is contained in $D^{*}$, differs from $d_{0}$ by an amount of order $O\left(\varrho^{4}\right)$. 
Our aim now is to calculate the univalent function $f^{*}(z)$ which maps $|z|<1$ onto $D^{*}$ with $f^{*}(0)=0$. In order to do this we first calculate the Green's function $g^{*}(w)=g^{*}(w, 0)$ of $D^{*}$ with the logarithmic pole at $w^{*}=0$.

Let $z=\varphi(w)$ be the inverse function of $w=f(z), g(w, \omega)$ be the Green's function of $D$ with the logarithmic pole of $\omega$, and $p(w, \omega)$ the analytic function in $w$ whose real part is $g(w, \omega)$. Using the general variational formula as developed in [6] we get (on denoting $g(w)=g(w, 0)$, $\left.p(t)=p(t, 0), \quad p^{\prime}(t, w)=\partial p(t, w) / \partial t\right)$

$$
g^{*}(w)=g(w)+\operatorname{Re}\left\{\frac{\varrho^{2}}{2 \pi i} \int_{\Gamma} p^{\prime}(t, w) p^{\prime}(t) v(t) d t\right\}+O\left(\varrho^{4}\right)
$$

where

$$
v(t)=\frac{a t}{t-w_{1}}+\frac{\bar{a} t^{2}}{\bar{w}_{1}\left(t-w_{1}^{\prime}\right)} ; \quad w_{1}^{\prime}=\frac{d_{0}^{2}}{\bar{w}_{1}}
$$

and $\Gamma$ is an arbitrary smooth closed curve such that $v(t)$ is regular in the closed domain bounded by $\Gamma$ and $C$.

Taking in account the formulas

$$
p(w)=-\log \varphi(w)
$$

and

$$
p(w, \omega)=\log \frac{1-\varphi(w) \overline{\varphi(\omega)}}{\varphi(w)-\varphi(\omega)}
$$

we obtain from (9) by the calculus of residues, on the assumption that $w_{1}, w_{1}^{\prime} \in D$ (which is always possible to choose when $d_{0}<1$ ),

$$
\begin{aligned}
g^{*}(w) & =g(w) \\
+\operatorname{Re} a \varrho^{2}\left[\frac{w \varphi^{\prime}(w)}{\varphi(w)} \frac{1}{w-w_{1}}\right. & +\frac{w_{1} \varphi^{\prime}\left(w_{1}\right)^{2}}{\varphi\left(w_{1}\right)} \frac{1}{\varphi\left(w_{1}\right)-\varphi(w)} \\
& \left.+\frac{\bar{w}_{1}^{\prime 2} \overline{\varphi^{\prime}\left(w_{1}^{\prime}\right)^{2}}}{w_{1} \overline{\varphi\left(w_{1}^{\prime}\right)}} \frac{\varphi(w)}{1-\overline{\varphi\left(w_{1}^{\prime}\right)} \varphi(w)}\right] \\
+\bar{a} \varrho^{2}\left[\frac{w^{2} \varphi^{\prime}(w)}{\bar{w}_{1} \varphi(w)} \frac{1}{w-w_{1}^{\prime}}+\right. & \frac{w_{1}^{\prime 2} \varphi^{\prime}\left(w_{1}^{\prime}\right)^{2}}{\bar{w}_{1} \varphi\left(w_{1}^{\prime}\right)} \frac{1}{\varphi\left(w_{1}^{\prime}\right)-\varphi(w)} \\
& \left.+\frac{\bar{w}_{1} \frac{1}{\varphi^{\prime}\left(w_{1}\right)^{2}}}{\overline{\varphi\left(w_{1}\right)}} \frac{\varphi(w)}{1-\overline{\varphi\left(w_{1}\right)} \varphi(w)}\right]+O\left(\varrho^{4}\right) .
\end{aligned}
$$


Completing the harmonic functions in (13) to analytic ones we get

$$
\begin{aligned}
& p^{*}(w)=p(w) \\
& +a \varrho^{2}\left[\frac{w \varphi^{\prime}(w)}{\varphi(w)} \frac{1}{w-w_{1}}+\frac{w_{1} \varphi^{\prime}\left(w_{1}\right)^{2}}{\varphi\left(w_{1}\right)} \frac{1}{\varphi\left(w_{1}\right)-\varphi(w)}\right. \\
& \left.+\frac{\bar{w}_{1}^{\prime 2} \overline{\varphi^{\prime}\left(w_{1}^{\prime}\right)^{2}}}{w_{1} \overline{\varphi\left(w_{1}^{\prime}\right)}} \frac{\varphi(w)}{1-\overline{\varphi\left(w_{1}^{\prime}\right)} \varphi(w)}\right] \\
& +\bar{a} \varrho^{2}\left[\frac{w^{2} \varphi^{\prime}(w)}{\bar{w}_{1} \varphi(w)} \frac{1}{w-w_{1}^{\prime}}+\frac{w_{1}^{\prime 2} \varphi^{\prime}\left(w_{1}^{\prime}\right)^{2}}{\bar{w}_{1} \varphi\left(w_{1}^{\prime}\right)} \frac{1}{\varphi\left(w_{1}^{\prime}\right)-\varphi(w)}\right. \\
& \left.+\frac{\bar{w}_{1} \overline{\varphi^{\prime}\left(w_{1}\right)^{2}}}{\overline{\varphi\left(w_{1}\right)}} \frac{\varphi(w)}{1-\overline{\varphi\left(w_{1}\right)} \varphi(w)}\right]+O\left(\varrho^{4}\right),
\end{aligned}
$$

or

$$
p^{*}(w)=p(w)+a \varrho^{2} M+\bar{a} \varrho^{2} N+O\left(\varrho^{4}\right)
$$

where $M$ and $N$ stand for the expression multiplying $a \varrho^{2}$ and $\bar{a} \varrho^{2}$, respectively, in (14).

From (15)

$$
\log \varphi^{*}(w)=\log \varphi(w)-a \varrho^{2} M-\bar{a} \varrho^{2} N+O\left(\varrho^{4}\right) .
$$

Taking exponents on both sides of this equation we arrive at

$$
\varphi^{*}(w)=\varphi(w)\left(1-a \varrho^{2} M-\bar{a} \varrho^{2} N\right)+O\left(\varrho^{4}\right) .
$$

Since

$$
z=\varphi(w), \quad \varphi^{\prime}(w)=\frac{1}{f^{\prime}(z)}, \quad w=f(z)
$$

we can express the right hand side of (17) in terms of $z$ and $f(z)$.

Furthermore, on observing that

$$
w=f^{*}\left(\varphi^{*}(w)\right)=f(\varphi(w))=f(z),
$$

we get from (17) that

(20) $f^{*}(z)=f(z)$

$$
\begin{aligned}
+a \varrho^{2}\left[\frac{f(z)}{f(z)-w_{1}}+z f^{\prime}(z)\left(\frac{w_{1} \varphi^{\prime}\left(w_{1}\right)^{2}}{\varphi\left(w_{1}\right)}\right.\right. & \frac{1}{\varphi\left(w_{1}\right)-z} \\
+ & \left.\left.\frac{\bar{w}_{1}^{\prime} \overline{\varphi^{\prime}\left(w_{1}^{\prime}\right)^{2}}}{w_{1} \overline{\varphi\left(w_{1}^{\prime}\right)}} \overline{1-\overline{\varphi\left(w_{1}^{\prime}\right)} z}\right)\right]
\end{aligned}
$$




$$
\begin{gathered}
+\bar{a} \varrho^{2}\left[\frac{f(z)^{2}}{\bar{w}_{1}\left(f(z)-w_{1}^{\prime}\right)}+z f^{\prime}(z)\left(\frac{w_{1}^{\prime 2} \varphi^{\prime}\left(w_{1}^{\prime}\right)^{2}}{\bar{w}_{1} \varphi\left(w_{1}^{\prime}\right)} \frac{1}{\varphi\left(w_{1}^{\prime}\right)-z}\right.\right. \\
\left.\left.+\frac{\bar{w}_{1} \overline{\varphi^{\prime}\left(w_{1}\right)^{2}}}{\overline{\varphi\left(w_{1}\right)}} \frac{z}{1-\overline{\varphi\left(w_{1}\right)} z}\right)\right]+O\left(\varrho^{4}\right) \\
=a_{1}^{*} z+a_{2}^{*} z^{2}+\ldots+a_{n}^{*} z^{n}+\ldots
\end{gathered}
$$

From (20) it follows that

$$
a_{1}^{*}=1+a \varrho^{2}\left[-\frac{1}{w_{1}}+\frac{w_{1} \varphi^{\prime}\left(w_{1}\right)^{2}}{\varphi\left(w_{1}\right)^{2}}\right]+\bar{a} \varrho^{2} \frac{w_{1}^{\prime 2}}{\bar{w}_{1}} \frac{\varphi^{\prime}\left(w_{1}^{\prime}\right)^{2}}{\varphi\left(w_{1}^{\prime}\right)^{2}}+O\left(\varrho^{4}\right)
$$

and

$$
\begin{aligned}
a_{n}^{*}= & a_{n}+a \varrho^{2}\left[-\frac{1}{w_{1}}\left(P_{n}\left(\frac{1}{w}\right)+a_{n}\right)\right. \\
& \left.+\frac{w_{1} \varphi^{\prime}\left(w_{1}\right)^{2}}{\varphi\left(w_{1}\right)^{2}} \sum_{k=1}^{n} \frac{k a_{n}}{\varphi\left(w_{1}\right)^{n-k}}+\frac{\bar{w}_{1}^{\prime 2} \overline{\varphi^{\prime}\left(w_{1}^{\prime}\right)^{2}}}{w_{1} \overline{\varphi\left(w_{1}^{\prime}\right)^{2}}} \sum_{k=1}^{n-1} k a_{k} \overline{\varphi\left(w_{1}^{\prime}\right)^{n-k}}\right] \\
+ & \bar{a} \varrho^{2}\left[-\frac{1}{\bar{w}_{1}} P_{n}\left(\frac{1}{w_{1}^{\prime}}\right)+\frac{w_{1}^{\prime 2} \varphi^{\prime}\left(w_{1}^{\prime}\right)^{2}}{\bar{w}_{1} \varphi\left(w_{1}^{\prime}\right)^{2}} \sum_{k=1}^{n} \frac{k a_{k}}{\varphi\left(w_{1}^{\prime}\right)^{n-k}}\right. \\
& \left.\quad+\frac{\bar{w}_{1} \overline{\varphi^{\prime}\left(w_{1}\right)^{2}}}{\overline{\varphi\left(w_{1}\right)^{2}}} \sum_{k=1}^{n-1} k a_{k} \overline{\varphi\left(w_{1}\right)^{n-k}}\right]+O\left(\varrho^{4}\right),
\end{aligned}
$$

where $P_{n}(x)$ is the polynomial given by the generating formula (see [7])

$$
\frac{f(z)}{1-x f(z)}=\sum_{k=1}^{\infty}\left[a_{k}+P_{k}(x)\right] z^{k} .
$$

Normalizing $f^{*}(z)$, we consider

$$
\tilde{f}(z)=\frac{f^{*}(z)}{a_{1}^{*}}=z+\tilde{a}_{2} z^{2}+\ldots+\tilde{a}_{n} z^{n}+\ldots
$$

where

$$
\begin{aligned}
\tilde{a}_{n}=a_{n} & +\frac{a \varrho^{2}}{w_{1}}\left[-P_{n}\left(\frac{1}{w_{1}}\right)+\frac{w_{1}^{2} \varphi^{\prime}\left(w_{1}\right)^{2}}{\varphi\left(w_{1}\right)^{2}}\left(\sum_{k=1}^{n-1} \frac{k a_{k}}{\varphi\left(w_{1}\right)^{n-k}}+(n-1) a_{n}\right)\right. \\
& \left.+\frac{w_{1}^{\prime 2} \varphi^{\prime}\left(w_{1}^{\prime}\right)^{2}}{\varphi-1} \sum_{k=1}^{n-1} k a_{k} \overline{\varphi\left(w_{1}^{\prime}\right)^{n-k}}\right] \\
+ & \frac{\bar{a} \varrho^{2}}{\overline{w_{1}}}\left[-P_{n}\left(\frac{1}{w_{1}^{\prime}}\right)+\frac{w_{1}^{\prime 2} \varphi^{\prime}\left(w_{1}^{\prime}\right)^{2}}{\varphi\left(w_{1}^{\prime}\right)^{2}}\left(\sum_{k=1}^{n-1} \frac{k a_{k}}{\varphi\left(w_{1}^{\prime}\right)^{n-k}}+(n-1) a_{n}\right)\right. \\
& \left.+\frac{\overline{w_{1}^{2}} \overline{\varphi^{\prime}\left(w_{1}\right)^{2}}}{\overline{\varphi\left(w_{1}\right)^{2}}} \sum_{k=1}^{n-1} k a_{k} \overline{\varphi\left(w_{1}\right)^{n-k}}\right]+O\left(\varrho^{4}\right) .
\end{aligned}
$$


Observe that $\tilde{f}(z) \in S(\tilde{d})$ where

$$
\begin{aligned}
\tilde{d} & =\left|\frac{d_{0}\left(1+O\left(\varrho^{4}\right)\right)}{\overline{\bar{a}} \varrho^{2}} \frac{a \varrho_{1}^{2} \varphi^{2}\left(w_{1}^{\prime}\right)^{2}}{w_{1}}\left(\frac{w_{1}^{2} \varphi^{\prime}\left(w_{1}\right)^{2}}{\varphi\left(w_{1}\right)^{2}}-1\right)+O\left(\varrho^{4}\right)\right| \\
& =d_{0}\left|1-\frac{\left.a \varrho^{\prime}\right)^{2}}{w_{1}}\left(\frac{w_{1}^{2} \varphi^{\prime}\left(w_{1}\right)^{2}}{\varphi\left(w_{1}\right)^{2}}-1\right)-\frac{\bar{a} \varrho^{2}}{\bar{w}_{1}} \frac{w_{1}^{\prime 2} \varphi^{\prime}\left(w_{1}^{\prime}\right)^{2}}{\varphi\left(w_{1}^{\prime}\right)^{2}}\right|+O\left(\varrho^{4}\right) .
\end{aligned}
$$

If $\varrho$ is sufficiently small

$$
\left|\tilde{d}-d_{0}\right|<\eta
$$

and therefore, because of the assumed local maximum property at $d_{0}$, it follows that

$$
\begin{aligned}
a_{n} d_{0}^{\lambda} \geqq & \left|\tilde{a}_{n}\right| \tilde{d}^{\lambda}=\mid a_{n}+\frac{a \varrho^{2}}{w_{1}}\left[-P_{n}\left(\frac{1}{w_{1}}\right)+\lambda a_{n}\right. \\
+ & \frac{w_{1}^{2} \varphi^{\prime}\left(w_{1}\right)^{2}}{\varphi\left(w_{1}\right)^{2}}\left(\sum_{k=1}^{n-1} \frac{k a_{k}}{\varphi\left(w_{1}\right)^{n-k}}+(n-1-\lambda) a_{n}\right) \\
& \left.\quad+\frac{\overline{w_{1}^{\prime}} \overline{\varphi^{\prime}\left(w_{1}^{\prime}\right)^{2}}}{\overline{\varphi\left(w_{1}^{\prime}\right)^{2}}} \sum_{k=1}^{n-1} k a_{k} \overline{\varphi\left(w_{1}^{\prime}\right)^{n-k}}\right] \\
+ & \frac{\bar{a} \varrho^{2}}{\bar{w}_{1}}\left[-P_{n}\left(\frac{1}{w_{1}^{\prime}}\right)+\frac{w_{1}^{\prime 2} \varphi^{\prime}\left(w_{1}^{\prime}\right)^{2}}{\varphi\left(w_{1}^{\prime}\right)^{2}}\left(\sum_{k=1}^{n-1} \frac{k a_{k}}{\varphi\left(w_{1}^{\prime}\right)^{n-k}}\right.\right. \\
+ & \left.\left.(n-1-\lambda) a_{n}\right)+\frac{\bar{w}_{1}^{2} \overline{\varphi^{\prime}\left(w_{1}\right)^{2}}}{\overline{\varphi\left(w_{1}\right)^{2}}} \sum_{k=1}^{n-1} k a_{k} \overline{\varphi\left(w_{1}\right)^{n-k}}\right] \\
& +O\left(\varrho^{4}\right) \mid d_{0}^{\lambda} .
\end{aligned}
$$

Hence, taking in account that $\operatorname{Re}\{x\}=\operatorname{Re}\{\bar{x}\}$, we get that

$$
\begin{aligned}
& \operatorname{Re}\left\{\frac { a \varrho ^ { 2 } } { w _ { 1 } } \left[\lambda a_{n}-P_{n}\left(\frac{1}{w_{1}}\right)\right.\right. \\
& +\frac{w_{1}^{2} \varphi^{\prime}\left(w_{1}\right)^{2}}{\varphi\left(w_{1}\right)^{2}}\left(\sum_{k=1}^{n-1}\left(\frac{k a_{k}}{\varphi\left(w_{1}\right)^{n-k}}+k \bar{a}_{k} \varphi\left(w_{1}\right)^{n-k}\right)+(n-1-\lambda) a_{n}\right) \\
& -\overline{P_{n}\left(\frac{1}{w_{1}^{\prime}}\right)}+\frac{\bar{w}_{1}^{\prime 2} \overline{\varphi^{\prime}\left(w_{1}^{\prime}\right)^{2}}}{\overline{\varphi\left(w_{1}^{\prime}\right)^{2}}}\left(\sum_{k=1}^{n-1}\left(\frac{k \bar{a}_{k}}{\overline{\varphi\left(w_{1}^{\prime}\right)^{n-k}}}+k a_{k} \overline{\varphi\left(w_{1}^{\prime}\right)^{n-k}}\right)\right. \\
& \left.\left.\left.+(n-1-\lambda) a_{n}\right)\right]+O\left(\varrho^{4}\right)\right\} \leqq 0
\end{aligned}
$$

must hold. 
Dividing by $\varrho^{2}$, letting $\varrho \rightarrow 0$ and taking in account that the argument of $a$ is arbitrary we end up with

$$
\begin{aligned}
\lambda & a_{n}-P_{n}\left(\frac{1}{w_{1}}\right) \\
& +\frac{w_{1}^{2} \varphi^{\prime}\left(w_{1}\right)^{2}}{\varphi\left(w_{1}\right)^{2}}\left[\sum_{k=1}^{n-1}\left(\frac{k a_{k}}{\varphi\left(w_{1}\right)^{n-k}}+k \bar{a}_{k} \varphi\left(w_{1}\right)^{n-k}\right)+(n-1-\lambda) a_{n}\right] \\
& +\left\{-P_{n}\left(\frac{1}{w_{1}^{\prime}}\right)\right. \\
& \left.+\frac{w_{1}^{\prime 2} \varphi^{\prime}\left(w_{1}^{\prime}\right)^{2}}{\varphi\left(w_{1}^{\prime}\right)^{2}} \sum_{k=1}^{n-1}\left(\frac{k a_{k}}{\varphi\left(w_{1}^{\prime}\right)^{n-k}}+k \bar{a}_{k} \varphi\left(w_{1}^{\prime}\right)^{n-k}+(n-1-\lambda) a_{n}\right)\right\}=0 .
\end{aligned}
$$

Formula (30) was proved for $w_{1} \in D$ with $w_{1}^{\prime} \in D$, otherwise arbitrary. Letting now $w_{1}^{\prime}$ tend to the boundary of $D,(30)$ will still hold. This shows that the function $f(z)$ is also analytic on $|z|=1$, and it maps the unit circle on analytic curves.

Denoting by

$$
\begin{aligned}
& M(w, \lambda)= \\
& \frac{w^{2} \varphi^{\prime}(w)^{2}}{\varphi(w)^{2}}\left[\sum_{k=1}^{n-1}\left(\frac{k a_{k}}{\varphi(w)^{n-k}}+k \bar{a}_{k} \varphi(w)^{n-k}\right)+(n-1-\lambda) a_{n}\right]-P_{n}\left(\frac{1}{w}\right)
\end{aligned}
$$

we get that the extremal function $w=f(z)$ satisfies the functional equation (written in terms of the inverse function $z=\varphi(w)$ )

$$
M(w, \lambda)+\overline{M\left(w^{\prime}, \lambda\right)}+\lambda a_{n}=0 .
$$

3. The domain $D$ has no exterior points. As mentioned above the extremal function $f(z)$ is analytic on $|z|=1$, except for some isolated singularities. We show now that the image domain has no exterior points.

Indeed, suppose to the contrary, that our assertion is incorrect. Then, there must exist an arc on $C$, in $|w|>d_{0}$, whose neighborhood does not completely belong to $D$. Let this arc (or a part of it) be the image of an arc on $|z|=1, \quad \vartheta_{1} \leqq \arg z \leqq \vartheta_{2}, \quad 0 \leqq \vartheta_{1}<\vartheta_{2}<2 \pi$ on which $f(z)$ is analytic.

Consider the domain $T$ whose boundary consists of $|z|=1, \vartheta_{2} \leqq$ $\arg z \leqq \vartheta_{1}+2 \pi$, and an arc given by $r=1+\varepsilon p(\vartheta)$, where $\varepsilon>0$ and sufficiently small and $p(\vartheta)$ satisfies

$$
p\left(\vartheta_{1}\right)=p\left(\vartheta_{2}\right)=0, \quad p(\vartheta)>0 \text { for } \vartheta_{1}<\vartheta<\vartheta_{2} .
$$


The function

$$
t(z)=z+\frac{\varepsilon z}{2 \pi} \int_{\vartheta_{1}}^{\vartheta_{3}} \frac{1+z e^{-i \vartheta}}{1-z e^{-i \vartheta}} p(\vartheta) d \vartheta+o(\varepsilon)
$$

maps $|z|<1$ onto $T$ and the mapping is univalent (see for example [4]). By our assumptions, the function $f(t(z))$ will be regular and univalent for sufficiently small $\varepsilon$, and the image domain will contain $|w|<d_{0}$.

In the development around $z=0$ of

$$
F(z)=f(t(z))=f\left(z+\frac{\varepsilon z}{2 \pi} \int_{\vartheta_{1}}^{\vartheta_{3}} \frac{1+z e^{-i \vartheta}}{1-z e^{-i \vartheta}} p(\vartheta) d \vartheta+o(\varepsilon)\right)
$$

the first coefficient is different from one. Therefore $F(z)$ does not belong to $S(d)$.

In order to obtain a near by function to the extremal one in $S(d)$ we make use of the following auxiliary function.

Let

$$
\begin{aligned}
\frac{s(z)}{\left(1+s(z) e^{-i \varphi}\right)^{2}}=\frac{4}{(1+\gamma)^{2}} \frac{z}{\left(1+z e^{-i \varphi}\right)^{2}}, \quad 0<\gamma=1-\delta<1, \\
0 \leqq \varphi<2 \pi .
\end{aligned}
$$

The function $s(z)$ maps $|z|<1$ onto $|s|<1$ with the radial slit from $\delta e^{i \varphi}$ to $e^{i \varphi}$. Taking $\delta>0$ sufficiently small we get from (36) that

$$
s(z)=z-\frac{\delta^{2}}{4} z \frac{1+z e^{-\imath \varphi}}{1-z e^{-i \varphi}}+o\left(\delta^{2}\right) .
$$

Choose $\varphi$ outside $\left[\vartheta_{1}, \vartheta_{2}\right]$, very close to $\vartheta_{1}$ or $\vartheta_{2}$, and consider the function

$$
\begin{aligned}
& F^{*}(z)=F(s(z))=f\left(s(z)+\frac{\varepsilon s(z)}{2 \pi} \int_{\vartheta_{1}}^{\vartheta_{2}} \frac{1+s(z) e^{-i \vartheta}}{1-s(z) e^{-i \vartheta}} p(\vartheta) d \vartheta+o(\varepsilon)\right) \\
& =f\left(z-\frac{\delta^{2}}{4} z \frac{1+z e^{-i \varphi}}{1-z e^{-i \varphi}}+\frac{\varepsilon z}{2 \pi} \int_{\vartheta_{1}}^{\vartheta_{2}} \frac{1+z e^{-i \vartheta}}{1-z e^{-i \vartheta}} p(\vartheta) d \vartheta+o(\varepsilon)+o\left(\delta^{2}\right)\right) .
\end{aligned}
$$

Choosing

$$
\frac{\delta^{2}}{4}=\frac{\varepsilon}{2 \pi} \int_{\vartheta_{1}}^{\vartheta_{2}} p(\vartheta) d \vartheta+o(\varepsilon)
$$


we get that

$$
F^{*}(z)=z+a_{2}^{*} z^{2}+\ldots+a_{n}^{*} z^{n}+\ldots
$$

where

$$
\begin{aligned}
& a_{n}^{*}=a_{n}+\frac{\varepsilon}{\pi} \int_{\vartheta_{1}}^{\vartheta_{2}}\left[(n-1) a_{n-1} e^{-i \vartheta}+\ldots+2 a_{2} e^{-(n-2) i \vartheta}+e^{-(n-1) i \vartheta}\right] p(\vartheta) d \vartheta \\
& -\frac{\varepsilon}{\pi} \int_{\vartheta_{1}}^{\vartheta_{2}}\left[(n-1) a_{n-1} e^{-i \varphi}+\ldots+2 a_{2} e^{-(n-2) i \varphi}+e^{-(n-1) i \varphi}\right] p(\vartheta) d \vartheta+o(\varepsilon),
\end{aligned}
$$

or

$$
\operatorname{Re} a_{n}^{*}=a_{n}+\frac{\varepsilon}{2 \pi} \int_{\vartheta_{1}}^{\vartheta_{2}}\left[Q\left(e^{i \vartheta}\right)-Q\left(e^{i \varphi}\right)\right] p(\vartheta) d \vartheta+o(\varepsilon)
$$

where

$$
Q(\xi)=\frac{1}{\xi^{n-1}}+\frac{2 a_{2}}{\xi^{n-2}}+\ldots+\frac{(n-1) a_{n-1}}{\xi}+(n-1) \bar{a}_{n-1} \xi+\ldots+\xi^{n-1}
$$

As $Q(\xi) \not \equiv 0$ on $|\xi|=1$ we may assume that $Q\left(e^{i \varphi}\right)$ is monotone and of constant sign in $\left[\vartheta_{1}, \vartheta_{2}\right]$. (If necessary we take a part of this interval to begin with.) If it is monotone increasing we choose $\varphi$ outside $\left[\vartheta_{1}, \vartheta_{2}\right]$ near $\vartheta_{1}$ and if $Q\left(e^{i \vartheta}\right)$ is monotone decreasing we take $\varphi$ near $\vartheta_{2}$.

By doing this we get from (42) that

$$
\operatorname{Re} a_{n}^{*}>a_{n}
$$

in contradiction to the extremal property of $a_{n}=a_{n}\left(d_{0}\right)$, and so we have proved our assertion that $D$ has no exterior points.

Next we prove the following

$\mathrm{L}$ e m m a. Let $a_{n}(d) d^{\lambda}$ have a local maximum at $d=d_{0}, \quad \lambda \geqq 0$. Then for every point $e^{i \varphi}$ whose image by the extremal function $f(z)=$ $z+a_{2} z^{2}+\ldots+a_{n} z^{n}+\ldots, \quad a_{n}=a_{n}(d)$, is at a distance from $w=0$ greater than $d_{0}$,

$$
\operatorname{Re}\left\{(n-1-\lambda) a_{n}+2(n-1) a_{n-1} e^{-i \varphi}+\ldots+2 e^{-(n-1) i \varphi}\right\} \geqq 0
$$

must hold, and equality occurs at points on $|z|=1$ whose images are tips of slits.

Proof. Let $e^{i \varphi}$ be such that $\left|f\left(e^{i \varphi}\right)\right|>d_{0}$. We consider the function 


$$
\tilde{f}(z)=\frac{f(s(z))}{1-\frac{\delta^{2}}{4}+o\left(\delta^{2}\right)}=z+\tilde{a}_{2} z^{2}+\ldots+\tilde{a}_{n} z^{n}+\ldots
$$

where $s(z)$ is the auxiliary function introduced in (36). This function belongs to $S(\tilde{d})$ where

$$
\tilde{d}=d_{0}\left(1+\frac{\delta^{2}}{4}+o\left(\delta^{2}\right)\right) .
$$

By choosing $\delta$ sufficiently small

$$
\tilde{d}=d_{0}+h, \quad 0<h<\eta .
$$

Hence

$$
\left|\tilde{a}_{n}\right| \tilde{d}^{\lambda} \leqq a_{n} d_{0}^{\lambda}
$$

As

$$
\tilde{a}_{n}=a_{n}-\frac{\delta^{2}}{4}\left((n-1) a_{n}+2(n-1) a_{n-1} e^{-i \varphi}+\ldots+2 e^{-(n-1) i \varphi}\right)+o\left(\delta^{2}\right),
$$

(47), (49) and (50) give (45).

We note that by letting $e^{i \varphi}$ approach $e^{i \varphi_{0}}$, whose image is a tip of a slit, we conclude that (45) holds also for $e^{i \varphi_{0}}$.

Let now $w_{0}$ be a tip of a slit, and consider a part of that slit that starts from $w_{0}$ not passing a forking point, if such a point exists. Let $w=w(t)$, $0 \leqq t \leqq t_{1}$, be its equation $\left(w(0)=w_{0}\right)$. By cutting out from the slit the portion from $w(0)$ to $w(t)$ we get a new domain in the $w$-plane. The function $f(z, t)$ which maps $|z|<1$ on this new domain is a solution of Loewner's differential equation [3]

$$
\frac{\partial f(z, t)}{\partial t}=z \frac{1+k(t) z}{1-k(t) z} \frac{\partial f(z, t)}{\partial z}
$$

where (by choosing the parameter $t$ in an appropriate manner)

$$
f(z, t)=e^{t} z+a_{2}(t) z^{2}+\ldots+a_{n}(t) z^{n}+\ldots
$$

with the initial condition

(53) $f(z, 0)=f(z)=z+a_{2}(0) z^{2}+\ldots+a_{n}(0) z^{n}+\ldots, \quad a_{n}(0)=a_{n}$

and $k(t)$ is a continuous function with $|k(t)|=1$. In addition the image of $1 / k(t)$ is the tip $w(t)$. 
From (51) and (52)

$$
\begin{gathered}
a_{n}^{\prime}(t)=2 e^{t} k(t)^{n-1}+2 a_{2}(t) k(t)^{n-2}+\ldots \\
+2(n-1) a_{n-1}(t) k(t)+n a_{n}(t) .
\end{gathered}
$$

Hence

(55) $a_{n}(t)=a_{n}+t\left(n a_{n}+2(n-1) a_{n-1} k(0)+\ldots+2 k(0)^{n-1}\right)+o(t)$,

where

$$
k(0)=e^{-i \varphi_{0}}
$$

The function

$$
F(z, t)=e^{-t} f(z, t)=z+\tilde{a}_{2}(t) z^{2}+\ldots+\tilde{a}_{n}(t) z^{n}+\ldots,
$$

where

(58) $\tilde{a}_{n}(t)=a_{n}+t\left((n-1) a_{n}+2(n-1) a_{n-1} k(0)+\ldots+2 k(0)^{n-1}\right)+o(t)$, belongs to $S\left(d_{1}\right)$ with

$$
d_{1} \geqq d_{0} e^{-t}
$$

By taking $t$ small enough

$$
d_{1}=d_{0}+h, \quad|h|<\eta \text {. }
$$

Hence, because of the local maximum at $d_{0}, \lambda \geqq 0$ and because of (59)

$$
\begin{aligned}
a_{n} d_{0}^{\lambda} \geqq\left|\tilde{a}_{n}(t)\right| d_{1}^{\lambda} \\
\geqq \mid a_{n}+t\left((n-1-\lambda) a_{n}+2(n-1) a_{n-1} e^{-i \varphi_{0}}+\ldots\right. \\
\left.+2 e^{-(n-1) i \varphi_{0}}\right)+o(t) \mid d_{0}^{\lambda} .
\end{aligned}
$$

From (61) it follows that

(62) $\operatorname{Re}\left\{(n-1-\lambda) a_{n}+2(n-1) a_{n-1} e^{-i \varphi_{0}}+\ldots+2 e^{-(n-1) i \varphi_{0}}\right\} \leqq 0$

must hold, (45) and (62) prove our assertion.

As a by-result from the lemma follows that a local maximum at a point $d_{0}$ can occur only with one exponent $\lambda=\lambda\left(d_{0}\right)$. We summarize the results obtained in the last two paragraphs in the following theorem

The or em 1. Let $a_{n}(d) d^{\lambda}$ have a local maximum at $d_{0}$ with $\lambda \geqq 0$, and let $w=f(z)=z+a_{2} z^{2}+\ldots+a_{n} z^{n}+\ldots, a_{n}=a_{n}\left(d_{0}\right)$, be an extremal function solving $\operatorname{Max}_{f \in S\left(d_{0}\right)}\left|a_{n}\right|$.

Then the extremal function maps $|z|<1$ on the whole w-plane slit along analytic slits and satisfies the functional equation

$$
M(w, \lambda)+\overline{M\left(w^{\prime}, \lambda\right)}+\lambda a_{n}=0
$$

where $M(w, \lambda)$ is given in (31). 
For every point $e^{i \varphi}$ whose image by the extremal functions has a distance from $w=0$ greater than $d_{0}$

$$
\operatorname{Re}\left\{(n-1-\lambda) a_{n}+2(n-1) a_{n-1} e^{-i \varphi}+\ldots+2 e^{-(n-1) i \varphi}\right\} \geqq 0
$$

with equality holding for those points on $|z|=1$ whose images are tips of the slits.

Moreover there is a unique non-negative exponent $\lambda$ for which there is a local maximum for $a_{n}(d) d^{\lambda}$ at $d_{0}$.

If the extremal function is such that it maps $|z|<1$ on the whole $w$-plane with slits which have only isolated points on $|w|=d_{0}$, then (64) will have a double zero at each point whose image is a tip of a slit situated on $|w|=d_{0}$. But then $M(w, \lambda)+\lambda a_{n} / 2$ will be regular on $|w|=d_{0}$, and, because of (63), will be pure imaginary there. Hence by the reflection principle will be regular in the whole $w$-plane, and therefore equal to a constant. Calculating the value of this expression at $w=0$ we find that it is equal to $-\lambda a_{n} / 2$, or that the extremal function satisfies the differential equation

$$
M(w, \lambda)+\lambda a_{n}=0
$$

which in the case of $\lambda=0$ is the Schiffer differential equation [6].

If the extremal function is such that it maps $|z|<1$ on the whole plane with slits such that one of them contains an arc of $|w|=d_{0}$ whose end points are $w_{1}, w_{2}\left(w_{1} \neq w_{2}\right)$ and the other slits have only isolated points on $|w|=d_{0}$ then

$$
\begin{array}{r}
\frac{\lambda a_{n}}{2}-\frac{1}{2}\left(P_{n}\left(\frac{1}{w}\right)+\overline{P_{n}\left(\frac{1}{w^{\prime}}\right)}\right)+\left[\sum_{k=1}^{n-1}\left(\frac{k a_{k}}{\varphi(w)^{n-k}}+k \bar{a}_{k} \varphi(w)^{n-k}\right)\right. \\
\left.+(n-1-\lambda) a_{n}\right] \frac{w^{2} \varphi^{\prime}(w)^{2}}{\varphi(w)^{2}}=N(w, \lambda)
\end{array}
$$

will be real on the arc on $|w|=d_{0}$ between $w_{1}$ and $w_{2}$ and will be pure imaginary outside this arc, on $|w|=d_{0}$.

Hence $N(w, \lambda)^{2}$ will be real on $|w|=d_{0}$ with simple poles at $w_{1}$ and $w_{2}$. Therefore the extremal function satisfies the differential equation

$$
N(w, \lambda)^{2}=\frac{A w^{2}+B w+C}{\left(w-w_{1}\right)\left(w-w_{2}\right)},
$$

with $A, B, C$, such that the expression on the right is real for $|w|=d_{0}$.

We summarize this in

Th e or e m 2. Let $d_{0}$ be a local maximum of $a_{n}(d) d^{\lambda}$. Then the extremal function maps $|z|<1$ on the whole w-plane slit along analytic slits. If no arc of $|w|=d_{0}$ is a part of the slits then the extremal function satisfies 
the differential equation $\left(65^{\prime}\right)$. If only one arc of $|w|=d_{0}$ is a part of some slit then the extremal function satisfies the differential equation $\left(67^{\prime}\right)$. Otherwise, at least two separate arcs on $|w|=d_{0}$ are parts of the slits.

4. The function $a_{n}(d)$. We prove now that $a_{n}(d), 1 / 4 \leqq d \leqq 1$, is a continuous function of $d$. Let $f(z)$ be an extremal function of $S(d)$ whose $n$th coefficient is $a_{n}(d)$. By considering the function introduced in (46) we get a function of $S(\tilde{d}), \tilde{d}=d\left(1+\delta^{2} / 4+o\left(\delta^{2}\right)\right)$, with the $n$th coefficient $\tilde{a}_{n}$ given by (50). So we constructed a function belonging to $S(\tilde{d}), \tilde{d}>d$, whose $n$th coefficient is arbitrary close to $a_{n}(d)$ if $\delta$ is chosen small enough.

In order to find a function belonging to $S(\tilde{d})$ with $1 / 4<\tilde{d}<d$ whose $n$th coefficient is arbitrary close to $a_{n}(d)$ if $d-\tilde{d}$ is small enough we proceed in the following way.

We first construct the function $\tilde{f}(z)$ introduced in (24). If for some choice of $a,|a|=1$, and $w_{1} \in D$ the coefficient $a_{1}^{*}$ from (21) is such that $\left|a_{1}^{*}\right|>1$ then our aim is achieved.

If however this is impossible then it follows that

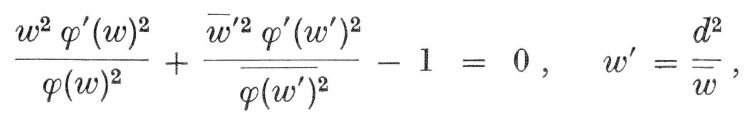

for every $w, w^{\prime} \in D$.

From (65) we also deduce that

$$
\frac{w^{2} \varphi^{\prime}(w)^{2}}{\varphi(w)^{2}}-\frac{1}{2}=\text { pure imaginary }
$$

on $|w|=d$, and hence the tips of the slits are on $|w|=d$.

If the number of slits is greater than one then by taking the function $F(z, t)$, introduced in (57), we obtain a function belonging to $S\left(d_{1}\right)$ with

$$
d_{1}=d e^{-t},
$$

which is smaller than $d$. By taking $t$ small enough, the $n$th coefficient $\tilde{a}_{n}(t)$ of this function, as given by (58), is very close to $a_{n}(d)$.

We now exclude the case that there is only one slit. Indeed, if such were the case we would have gotten from (66), by the reflection principle, that

$$
\frac{w^{2} \varphi^{\prime}(w)^{2}}{\varphi(w)^{2}}-\frac{1}{2}=\frac{A w+B}{w-w_{0}}
$$


where $\left|w_{0}\right|=d$ and $(A w+B) /\left(w-w_{0}\right)$ is pure imaginary on $|w|=d$. By substituting $w=0$ and $w=\infty$ in (68) we get $B=w_{0} / 2$ and $A=-1 / 2$.

Finally

$$
\frac{w^{2} \varphi^{\prime}(w)^{2}}{\varphi(w)^{2}}=\frac{w_{0}}{w_{0}-w} .
$$

Solving this differential equation we get that

$$
w=f(z)=z\left(1+\frac{z}{4 w_{0}}\right)^{-2},
$$

and hence, because of the existence of a singularity on $|z|=1$,

$$
\left|4 w_{0}\right|=1
$$

must hold. But this is in contradiction to $\left|w_{0}\right|=d>1 / 4$.

The continuity of $a_{n}(d)$ follows now by arguments of normal families, and our assertion is established. At points of local maxima we can say more about $a_{n}(d)$. We prove the following

The or e m 3. Let $d_{0}$ be a point of local maximum for $a_{n}(d) d^{\lambda}$, $\lambda \geqq 0$. Then the function $a_{n}(d)$ has a right hand derivative at $d_{0}$, and if $\lambda>0$ it has a derivative there. The value in both cases is $\left(-\lambda / d_{0}\right) a_{n}\left(d_{0}\right)$.

Proof. From the local maximum at $d_{0}$ we have

$$
a_{n}\left(d_{0}+h\right)\left(d_{0}+h\right)^{\lambda} \leqq a_{n}\left(d_{0}\right) d_{0}^{\lambda}, \quad 0<h<\eta .
$$

Hence

$$
\limsup _{h \rightarrow 0+} \frac{\log a_{n}\left(d_{0}+h\right)-\log a_{n}\left(d_{0}\right)}{h} \leqq-\frac{\lambda}{d_{0}} .
$$

On the other hand for any $\varepsilon>0$ we have

$$
a_{n}\left(d_{0}+h\right)\left(d_{0}+h\right)^{\lambda+\varepsilon} \geqq a_{n}\left(d_{0}\right) d_{0}^{\lambda+\varepsilon}, \quad 0<h<\eta_{1} .
$$

This we see by aid of the functions $\tilde{f}(z)$ introduced in (46). Take $e^{i \varphi}$ very close to $e^{i \varphi_{0}}$, with $\left|f\left(e^{i \varphi}\right)\right|>d_{0}$, so that

$$
\operatorname{Re}\left\{(n-1-\lambda) a_{n}+2(n-1) a_{n-1} e^{-i \varphi}+\ldots+2 e^{-(n-1) i \varphi}\right\}<\varepsilon a_{n},
$$

(the left hand side of (75) though positive is very close to zero).

In accordance $\delta$ is taken small enough so that

$$
\tilde{d}=d_{0}\left(1+\frac{\delta^{2}}{4}+o\left(\delta^{2}\right)\right)=d_{0}+h .
$$

Using (50) we get that 
(77)

$$
\begin{aligned}
& a_{n}\left(d_{0}+h\right)\left(d_{0}+h\right)^{\lambda+\varepsilon} \geqq\left|\tilde{a}_{n}\right| \tilde{d}^{\lambda+\varepsilon} \\
= & \mid a_{n}-\frac{\delta^{2}}{4}\left[(n-1-\lambda-\varepsilon) a_{n}+2(n-1) a_{n-1} e^{-i \varphi}+\ldots+2 e^{-(n-1) i \varphi}\right] \\
& +o\left(\delta^{2}\right) \mid d_{0}^{\lambda+\varepsilon},
\end{aligned}
$$

and because of (75) the desired result (74) follows.

From (74) it follows that

$$
\liminf _{h \rightarrow 0+} \frac{\log a_{n}\left(d_{0}+h\right)-\log a_{n}\left(d_{0}\right)}{h} \geqq-\frac{\lambda+\varepsilon}{d_{0}} .
$$

As this last result holds for every $\varepsilon>0$, it holds also for $\varepsilon=0$. Together with (73) the assertion of the theorem follows.

Next, if $\lambda>0$, we show that also the left hand derivative exists and is again equal to $\left(-\lambda / d_{0}\right) a_{n}\left(d_{0}\right)$.

Take the function introduced in (57). This function $F(z, t) \in S\left(d_{1}\right)$ with $d_{1}$ satisfying (59). For any $\varepsilon>0, \lambda-\varepsilon>0$, we get, by using (58) and (59), that

$$
\begin{aligned}
a_{n}\left(d_{1}\right) d_{1}^{\lambda-\varepsilon} \geqq\left|\tilde{a}_{n}(t)\right|\left(d_{0} e^{-t}\right)^{\lambda-\varepsilon} & \\
=\mid a_{n}+t\left((n-1-\lambda+\varepsilon) a_{n}+2(n-1) a_{n-1} e^{-i \varphi_{0}}\right. & \left.+\ldots+2 e^{-(n-1) i \varphi_{0}}\right) \\
& +o(t) \mid d_{0}^{\lambda-\varepsilon}
\end{aligned}
$$

from which it follows that

$$
a_{n}\left(d_{1}\right) d_{1}^{\lambda-\varepsilon}>a_{n} d_{0}^{\lambda-\varepsilon} .
$$

By taking $t$ arbitrary small the value of $d_{1}$ is very close to $d_{0}$. Because of the local maximum property at $d_{0}$ and (80) it follows that

$$
d_{1}=d_{0}-h, \quad h>0 .
$$

It now follows from (80), (81) and

$$
a_{n}\left(d_{1}\right) d_{1}^{\lambda} \leqq a_{n} d_{0}^{\lambda}
$$

in a similar way to that used before for the right hand derivative, that the left hand one also exists and is again equal to $\left(-\lambda / d_{0}\right) a_{n}\left(d_{0}\right)$.

We conclude with the following remark. It is easy to see that $a_{2}(d)$ is a monotone decreasing function. Otherwise there would exist a local maximum at a point $d_{0}, d_{0}>1 / 4$, and the extremal function will satisfy the Schiffer differential equation. It will then follow, because of the double zero of $1 / z+a_{2}+z$ on $|z|=1$, that $a_{2}=a_{2}\left(d_{0}\right)=2$, and hence $d_{0}=1 / 4$ against our assumption . 
We conjecture that $a_{n}(d)$ is monotone decreasing for every $n$, from which the Bieberbach conjecture would have followed.

\section{References}

[1] Garabeidian, P. R., G. G. Ross, and M. M. SchifFer: On the Bieberbach conjecture for even $n$. - J. Math. Mech. 14, 1965, 975-989.

[2] Garabedian, P. R., and M. M. SchiffER: The local maximum theorem for the coefficients of univalent functions. - Arch. Rational Mech. Anal. $26,1967,1-32$.

[3] LOEWNER, C.: Untersuchungen über schlichte konforme Abbildungen des Einheitskreises, I. - Math. Ann. 89, 1923, 103-121.

[4] NeHARI, Z.: Conformal mapping. - McGraw Hill, New York-Toronto-London, 1952.

[5] NeTAnyahu, E.: On univalent functions in the unit disk whose image contains a given disk. - J. Analyse Math. 23, 1970, 305-322.

[6] SCHIFFER, M.: Hadamard's formula and the variation of domain functions. Amer. J. Math. 68, 1946, 417-448.

[7] -》- Faber polynomials in the theory of univalent functions. - Bull. Amer. Math. Soc. 54, 1948, 503-517.

Technion - Israel Institute of Technology

Department of Mathematics

Haifa

Israel

Received 15 August 1975
University of Illinois at Urbana-Champaign

Department of Mathematics

Urbana

Illinois 61801

USA 